\title{
Relationship Between Color Preference, Emotional Intelligence, and Interpersonal Relationship of Nursing Students
}

\author{
Gye-Hyun Jung ${ }^{1}$, Youn-Suk Ham ${ }^{2}$ and Do-Young Lee ${ }^{3 *}$ \\ ${ }^{1}$ Dept. of Nursing, Daejon University, Korea \\ 2,3*Dept. of Nursing, Ansan University, Korea \\ 1'ghjung@dju.ac.kr, ${ }^{2}$ hyhees67@ansan.ac.kr, ${ }^{3}{ }^{*}$ sunshinenurse@ hanmail.net
}

\begin{abstract}
The purpose of this study is to provide the basic data for the program to enhance the adaptability of the nursing department students by analyzing their color preference, emotional intelligence and the interpersonal relationship. The data were collected through the structured self-reported questionnaires and to verify the difference in the emotional intelligence and interpersonal relationship depending on the color preference, $t$-test, ANOVA, and the correlation were analyzed using the Pearson's correlation coefficients with the SPSS 20.0 program. In the results, the color preference of the nursing department students showed to have correlation with the emotional intelligence and the interpersonal relationship. Therefore, based on the results of this study, it is expected that the study on the color should be made continuously with the nursing department students so that the emotional intelligence and the interpersonal relationship can be improved, and would reflect their results actively.
\end{abstract}

Keywords: Color, Preference, Emotional, Intelligence, Interpersonal, Relationship, Nursing, Students

\section{Introduction}

In contemporary society, color is used in numerous ways and affects our overall life [1]. The colors felt by humans are a result of sensory reaction where light that comes in through the optic nerves are interpreted as colors rather than being something that physically exists [2]. While colors were used as a means to describe objects or distinguish people of different classes, today it use has been expanded to be applied to improve our physical and mental health [3].

The reason programs using color can affect our life and health is because colors can trigger psychological responses and changes in emotion, in addition to bringing about physical reactions. In recent years, many attempts and measures using color are being presented as a way to address tension and anxiety felt by contemporary people. Various programs using color in psychological counseling have contributed to improving our quality of life and health. This is because colors bring about psychological responses and emotional change, which in turn leads to physical responses.

Colors stimulate the emotions felt by people and emotions triggered by colors constantly change [4]. But the subjective preference of individuals for a certain color is referred to as color preference. The difference in color preference is due to the difference in expertise in

Article history:

Received (November 1, 2017), Review Result (December 26, 2017), Accepted (January 28, 2018) 
color, interest or temperament which leads to a difference in the degree of how color is recognized. Color preference may vary according to culture or environment, too. As such, understanding colors and color preference can be a way to understand individuals' psychological state. By verifying the preferred colors of people, we can apply the learning to their interpersonal issues or learning problems. Color intelligence is something that is more affected by learning rather than something one is born with. As such, appropriate emotional intelligence education is needed. For example, those who have undergone emotional education through art education are found to have a higher EQ [5]. Various factors affect interpersonal relationships. First, needing to understand his own emotions and express them appropriately are important [6].

As such, this study reviews the color preference of students majoring in nursing studies at universities and verifies the relation between EQ and interpersonal relationships in accordance with color preference to provide a basic set of data for the development of programs

\section{Methodology}

\subsection{Design of the study}

This is a descriptive study on the correlation between the variables of color preference, EQ and interpersonal relationships of nursing students.

\subsection{Study tools}

\subsubsection{Color preference}

To measure color preference, the eight colors used in [7]'s CRR, red, orange, yellow, green, turquoise, blue, purple and magenta were used. This is a tool that uses both color and form to have a complementary effect. To review the EQ in accordance with color preference, colors were divided into warm colors (red, orange, yellow), cold colors (blue, turquoise, navy) and neutral colors (green, purple, magenta) according to the classification by Munsell.

\subsubsection{Emotional intelligence}

To measure emotional intelligence, the translated version of the Wong and Law Emotional Intelligence Scale: WLEIS developed by [8] was used. WLEIS consists of a total of 16 questions with four questions on understanding one's own emotions, four questions on understanding other's emotions, four questions on emotional control and four questions on emotional application. The response options ranged from "very much so" which was given 7 points to "not at all" which was given 1 point, on a 7 point Likert scale. A higher score indicates higher emotional intelligence. At the time of development, confidence level had a Cronbach's $\alpha=.90$, with the confidence level for this study being .75 .

\subsubsection{Changes in relationships}

Relationships were measured using the Relationship Cahnge Scale developed by [9] and translated by Moon (1980). This tool measures the seven categories of satisfaction, communication, reliability, intimacy, openness, understanding and sensitivity using a total of 25 questions on a 5 point scale, with "not at all" given 1 point and "very much so" given 5 
points. A higher score indicates a more positive relationship. At the time of development, Cronbach's alpha was .86, while for this study it was .94 .

\section{Subjects}

The number of subjects was calculated using the $\mathrm{G}^{*}$ Power 3.1.9.2 program which is a program for calculating the number of samples in accordance with the formula of Cohen. When correlation analyses for three independent variables were to be used with a significance level of $5 \%$ and a statistical significance of $95 \%$, the minimum sample size was 185 . Considering a dropout rate of $15 \%$, it was decided on 250 subjects [10].

\section{Data collection and ethical considerations}

Data for the study was collected through two self-reporting structured questionnaires distributed to nursing students from June 17 to July 30, 2017. For ethical considerations, first the study was reviewed and approved by the life ethics committee at D University (approval number: 1040647-201706-HR-002-03.) After explaining the purpose of the study, the time it would take for the questionnaire and confidentiality of personal data to the professors of the university and the subjects, voluntary participation and consent took place. Nursing students who wished to take part signed a consent form and were told they could drop out of the study at any time. It was also explained that the data would not be used for any other purpose than the study and confidentiality would be preserved. The questionnaire took about 10-15 minutes.

\section{Statistical analysis}

The general characteristics of the study subjects were analyzed using actual figures, percentages, the mean and standard deviation.

Color preference, emotional intelligence and changes in relationships were analyzed using mean and standard deviation.

The difference in color preference, emotional intelligence and changes in relationships across different general characters were analyzed using a t-test and ANOVA, and a Scheffe test for post verification.

The correlation between color preference, emotional intelligence and changes in relationships was analyzed using Pearson's correlation coefficients.

\section{Findings}

\subsection{General characteristics of the subjects}

Of the total of 210 subjects, $91.9 \%$ (193 people) were female, and $8.1 \%$ (17 people) were male, with an average age of 24.38 years. Those between the ages 21 and 25 accounted for the largest share of $82.4 \%$ (173 people), while those aged 26 or over accounted for $17.6 \%$ (37 people). For religion, 31.4\% (66 people) were Christian and 15.7\% (33 people) were Catholic. In terms of siblings, those who were the eldest person accounted for the largest share at $46.7 \%$ (98 people), followed by the youngest among their siblings at $38.1 \%$ (80 people). In terms of color preference, blue was chosen the most by $21.4 \%$ (45 people), followed by yellow at $16.2 \%$ (34 people), magenta at $14.8 \%$ (31 people), red at $12.9 \%$ (27 people), green at $12.4 \%$ (26 people), purple at $9.5 \%$ (20 people), turquoise at $6.7 \%$ (14 people), and orange at $6.2 \%$ (13 people). 


\subsection{Variables of subjects}

The average score for emotional intelligence of the subjects was 82.98 points. In terms of the sub-factors, understanding of one's own emotions was highest at an average of 22.15 points, understanding of other's emotions was 21.72 points, emotional control 20.21 points and emotional application the lowest at an average of 18.88 points. The changes in relationships of the subjects was higher than average at a mean of 93.40 points. The subfactors of sensitivity was an average of 18.28 points, followed by understanding at 16.14 points, communication at 14.98 points, satisfaction at 14.41 points, intimacy at 11.68 points and reliability at 10.54 points.

\subsection{Differences in variables across different general characteristics of the subjects}

The differences in variables according to the general characteristics of the subjects are as follows. For emotional intelligence, there was significant difference for age $(\mathrm{F}=2.91, \mathrm{p}<0.001)$ and color preference $(\mathrm{F}=4.15, \mathrm{p}=0.017)$ but this was not statistically significant. But there was difference for gender $(\mathrm{t}=1.69, \mathrm{p}=0.091)$ and birth order among siblings $(\mathrm{F}=2.25, \mathrm{p}=0.083)$. A post-analysis revealed that subjects who preferred cold colors had a higher emotional intelligence than those who preferred warm colors. Changes in relationships had a significant difference across gender $(\mathrm{t}=3.41, \mathrm{p}=0.001$ and while there was no statistical significance, there was also difference in color preference $(\mathrm{F}=2.48, \mathrm{p}=0.086)$.

\section{Discussion}

This study was conducted to provide a basic set of data for the development of a program that can improve the adjustability of nursing students, by analyzing the correlation between the students' color preference, emotional intelligence and relationships. After conducting the study on 210 senior year students of nursing studies, it was found that there was a difference in emotional intelligence and changes in relationships in accordance with color preference. Color is something that one learns the meaning of, and thus its meaning changes with an individual's temperament or the times [11]. Color carries symbolism that stands for universal emotions. Certain color preferences are correlated with one's education environment, psychological effects from the surrounding environment, one's character and temperament [12].

In a study conducted on day care teachers to review the correlation between color reference and emotional intelligence, those who preferred warm colors were found to have the highest emotional intelligence while those who preferred cold colors had the lowest [13]. This is in line with preceding studies that found that those who like warm colors are more emotional than those who like cold colors [14]. Warm colors increase psychological energy and represent softness, warmth and friendliness. The study finding of [15], too, state that warm colors represent happy/ enjoyable, comfort/serenity and happiness, that children who frequently use warm colors express positive energies, and that warm colors express emotions.

As such, based on the findings of this study, we hope studies on color associated with nursing students will be continued and their results reflected on various programs to help students improve their emotional intelligence and relationships. 


\section{Suggestions}

This study was conducted on nursing students at two universities and therefore needs iteration with a larger number of subjects to verify the validity of the findings. There are subjective factors related to the perception and attitude towards color. Therefore in order to generalize the study findings on color, various factors need to be factored in. Continued management is also encouraged for the development of a clinical program that can help improve emotional intelligence and relationships using color.

\section{Acknowledgements}

The authors wish to thank everyone who contributed data to this study.

\section{References}

[1] T.H. Ha and H.K. Back, "A study on color meditation programs for wounded heart healing of university students,"Journal of Digital Convergence, vol.20, no.5, (2014)

[2] Y.S. Park, "Society·culture environment", Sallim books, Gyeonggi-do, Paju, (2004)

[3] J.H. Kim, "Color \& human response," Sigoogsa, Seoul, Seocho, (2010)

[4] J.M. Lee, "The power of color," Iljinsa, Seoul, Yong-San, (2013)

[5] Y.J. Lee, N.K. Lee, and S.J. Kim, "A comparison of color sensibility judgment in color professionals and non-professionals - focus on the respective contribution of hue and tone," Color new, vol.26, no.4, (2012)

[6] H.W. Lee, D.M. Yom, and M.H. Lee, "Impact of college students' mood awareness on interpersonal relationship: Focus on mediating effect of emotional expression," vol.30, no.2, (2014)

[7] S. Howard and S. Dorothy, "Color your life: How to use the right colors to achieve balance, health, and happiness," Tarcher Perigee, USA, (2013)

[8] C.S. Wong and S.L. Law, "The effect of leader and follower emotional intelligence on performance and attitude," Exploratory Study Leadership Quarterly, vol.13, no.3, (2002)

[9] A. Schlein, B.G. Guerney, "Relationship enhancement," Sanfrancisco, CA: Josey-Bass, (1971)

[10] F. Faul, E. Erdfelder, A. Lang, and A. Buchner, "G*Power 3: A flexible statistical power analysis for the social, behavioral, and biomedical sciences," Behavioral Research Methods, vol.39, no.2, pp.175-191, (2007)

[11] G.Y. Je and K.H. Lee, "Color preference and color meaning of university students," Journal of the Korean Society of Clothing Industry, vol.13, no.3, (2011)

[12] Y.S. Park and S.A. Song, "The research on preference colors and color image of the 20's," Journal of Korea Society of Color Studies, vol.28, no.3, (2014)

[13] J.H. Park and H.J. Ko, "Color preference and emotional intelligence of early childhood teachers," Korean Journal of Art Therapy, vol.21, no.6, (2014)

[14] Birren \& Faber, "Color perception in Art," Schiffer Publishing, (1976)

[15] Y.J. Jung, "The relationship between children's emotion and color and favorite colors," Korean Art Education Association, vol.22, no.1, (2008) 
Relationship Between Color Preference, Emotional Intelligence, and Interpersonal Relationship of Nursing Students

\section{Authors}

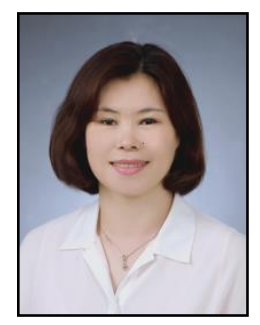

\section{Gye-Hyun Jung}

Ph.D., Dept. of Nursing, Graduate School of Daejon University

Assistant professor, Dept. of Nursing, Daejon University

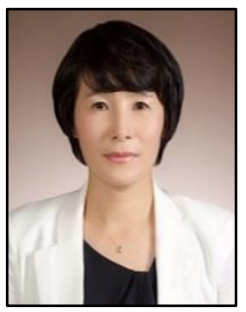

\section{Youn-Suk Ham}

Assistant professor, Dept. of Nursing, Ansan University

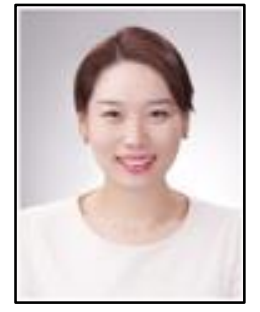

\section{Do-Young Lee}

Ph.D., Dept. of Nursing, Graduate School of Ajou University Assistant professor, Dept. of Nursing, Ansan University 